\title{
NY-BR-1 is a Differentiation Antigen of the Mammary Gland
}

\author{
Dirk Jäger MD,* Valeriy Filonenko, PhD, $\dagger$ Ivan Gout, PhD, $\dagger+$ Denise Frosina, BS, $\S$ \\ Susannah Eastlake-Wade, BA,§ Sandra Castelli, BS,§ Zsuzsanna Varga, MD,\| Holger Moch, MD,\| \\ Yao-Tseng Chen, MD, PhD, 9 Klaus J. Busam, MD,\# Inka Seil, PhD,** Lloyd J. Old, MD, \\ Aviram Nissan, MD, PhD, $\dagger+$ Claudia Frei, $+\uparrow$ Ali O. Gure, MD, PhD, $\S$ \\ Alexander Knuth, MD, $\neq$ t and Achim A. Jungbluth, MD§
}

\begin{abstract}
NY-BR-1 was recently identified by autologous serological typing of the recombinant expression library in a breast cancer patient. Extensive reverse transcriptase-polymerase chain reaction analysis revealed the presence of NY-BR-1 in normal breast tissue and tumors derived thereof. Except normal testis, no other normal tissue or tumors showed NY-BR-1 expression. However, nothing is known about the expression of its actual antigen. In the present study, we describe the generation of 2 new monoclonal antibodies, NY-BR-1\#2 and NY-BR-1\#3, to NY-BR-1 for the analysis of its expression on a protein level employing recombinant NY-BR-1 protein for the immunization of BALB/c mice. In normal tissues, immunohistochemical testing demonstrates NY-BR-1 in a mostly focal fashion in the epithelia of ducts and acini of the mammary gland. No other tissue was immunopositive including testis. In tumors, homogenous staining can be seen in almost all ductal carcinomas in situ and/or the intraductal component of invasive carcinomas. Invasive carcinomas show a lower number of NY-BR-1-positive tumors. Initial higher numbers of NY-BR-1 mRNA-positive invasive carcinomas are most likely based on sample error owing to the contamination of tumor tissue with remnants of normal breast epithelium. Sweat gland carcinomas, which are related to breast cancer, are also positive in about one-third of the cases. These
\end{abstract}

Received for publication November 30, 2005; accepted March 9, 2006. From the *Medizinische Onkologie NCT, University Hospital Heidelberg, 69120 Heidelberg; †The Laboratory of Cell Growth Regulation, Institute of Molecular Biology and Genetics, Kyiv, Ukraine; $\ddagger$ Department of Biochemistry and Molecular Biology, University College London, UK; §Ludwig Institute for Cancer Research at Memorial Sloan-Kettering Cancer Center; $\uparrow$ Weill Medical College of Cornell University; \#Department of Pathology, Memorial SloanKettering Cancer Center, New York, NY; \|Departments of Pathology; **Institute for Biomedical Research Georg-Speyer-Haus, Frankfurt, Germany; \$Oncology, University Hospital Zurich, Switzerland; and ††Laboratory of Surgical Oncology, Department of Surgery, Hadassah University Hospital Mount Scopus, Jerusalem, Israel.

Part of this work was presented at the 2006 annual meeting of the United States and Canadaian Academy of Pathology (USCAP) in Atlanta.

Reprints: Achim Jungbluth, MD, Ludwig Institute for Cancer Research, New York Branch, at Memorial Sloan-Kettering Cancer Center, 1275 York Avenue, Box 32, New York, NY 10021 (e-mail: jungblua@mskcc.org).

Copyright (C) 2007 by Lippincott Williams \& Wilkins data indicate that NY-BR-1 is a differentiation antigen of the mammary gland that could be useful for diagnosis and/or immunotherapy of breast carcinomas.

Key Words: NY-BR-1, breast cancer, breast differentiation antigen

(Appl Immunohistochem Mol Morphol 2007;15:77-83)

A $\mathrm{s}$ a result of an attempt to find new tumor-associated antigens suitable for immunotherapy approaches in cancer, $N Y-B R-1$ was recently identified by the serological analysis of recombinant cDNA expression library (SEREX) method employing the serological screening of a tumor-derived recombinant expression library with autologous serum of a breast cancer patient. ${ }^{1} N Y-B R-1$ consists of 37 exons encoding a protein of 150,000 to $160,000 \mathrm{kDa}$ and is localized on chromosome 10. Reverse transcriptasepolymerase chain reaction (RT-PCR) analyses revealed the presence of NY-BR-1 solely in the mammary gland and in testis but in no other normal organ. ${ }^{1,2}$ In tumors, NY-BR1 was expressed in a high number of breast carcinomas and not in any other neoplasm. Owing to this restricted expression pattern, NY-BR-1 seems to be a suitable target for the immunotherapy of breast cancer. However, current knowledge about NY-BR-1 expression is exclusively based on RT-PCR analyses and nothing is known about its presence on a protein level.

Consequently, in the present study we generated monoclonal antibodies (mAbs) to NY-BR-1 to analyze its expression on a protein level. Here, we show that the presence of actual antigen is restricted to normal as well as malignant breast (and related) epithelium and hence justifies the designation of NY-BR-1 as a differentiation antigen of the mammary gland.

\section{MATERIALS AND METHODS}

\section{Generation of Anti-NY-BR-1 mAbs}

\section{Plasmids and Recombinant Protein}

The putative complete NY-BR-1 cDNA is of $6465 \mathrm{bp}$, having a $5^{\prime}$-untranslated region of $168 \mathrm{bp}$, a 
coding region of $4194 \mathrm{bp}$, and a $3^{\prime}$-untranslated region of $2088 \mathrm{bp}$ excluding the poly-A tail. Two overlapping fragments of the coding sequence (bp1-2181 and bp2112-4191) were PCR amplified using normal testis cDNA and cloned into the PCR cloning vector pGEMT. Using a shared restriction site (Fsp1) of the overlapping fragments, the 2 fragments were ligated to a full-length NY-BR-1 (bp1-4191) construct. The full-length NY-BR-1 cDNA was subcloned into the pcDNA3.1 expression vector (Invitrogen) A similar NY-BR-1 construct carrying a C-terminal GFP cDNA (pcDNA3.1-NY-BR-1GFP) was also generated.

A NY-BR-1 cDNA fragment encoding aa 701-1397 was amplified from the full-length construct and cloned into the prokaryotic expression vector pQE30 (Qiagen, Valencia, CA). Competent M15 (pREP4) Escherichia coli were transformed and the recombinant protein was produced and purified under denaturing condition following the manufacturer's protocol. The purified recombinant His-tag protein was analyzed by sodium dodecyle sulphate-polyacrylamide gel electrophoresis, followed by silver staining.

For the generation of mAbs, a short $231 \mathrm{bp} \mathrm{NY}$ BR-1 cDNA fragment was amplified (forward primer GCGCATATGAAAGTTTCTATTCCAACT, reverse primer TGACTCGAGACTCTCAGAATCCCAAGA) encoding a 77-aa peptide (NY-BR-1 aa 851-928) having no homologies to other proteins deposited in the databases and cloned into the pET24a expression vector (Novagen, San Diego, CA) in frame with His-tag sequence using $N d e \mathrm{I}$ and XhoI restriction sites. The recombinant His-tagged protein was expressed in BL DE3 cells and purified from the cell lysate by Ni-NTA Sepharose chromatography according to the manufacturer's recommendation. Purified protein was used for the immunization of BALB/c mice and screening of positive hybridoma clones was carried out by enzyme-linked immunosorbant assay (ELISA) analysis.

\section{Generation of Anti-NY-BR-1 mAbs}

NY-BR-1-specific hybridoma cells were generated as described previously. ${ }^{3}$ Briefly, main steps included: (a) immunization of BALB/c mice with His-tagged NY-BR-1 protein; (b) culturing of BALB/c parental myeloma cells $\mathrm{SP} 2 / 0$; (c) preparation of splenocytes from immunized mice; (d) fusion of prepared splenocytes with myeloma cells using PEG 1500; and (e) selection of positive hybridoma clones by Western blot. Two anti-NY-BR-1specific clones, NY-BR-1\#2 and NY-BR-1\#3, were selected based on Western blotting and immunohistochemical (IHC) analysis. Newly generated hybridoma supernatants were screened by immunoblotting using $40 \mu \mathrm{g} / \mathrm{slot}$ whole-cell lysates from native and NY-BR-1 transfected $293 \mathrm{~T}$ cells and recombinant protein preparations of NY-BR-1-aa701-1397. The recombinant proteins NY-ESO-1, SSX-4, and MAGE-A10 were used as negative controls according to standard protocols as described previously. ${ }^{3,4}$ The 293 T-cell line was maintained under conditions recommended by the ATCC.
Cells were transfected with pcDNA3.1-NY-BR-1-aa 1-1397 and pcDNA-NY-BR-1-aa1-1397-GFP using Lipofectamine 2000 (Invitrogen, Carlsbad, CA) according to the manufacturer's instructions. For immunoblotting, lysates $(40 \mu \mathrm{g}$ protein) were separated on $6 \%$ polyacrylamide gels and transferred onto nitrocellulose membranes. Proteins were detected using supernatant of newly generated anti-NY-BR-1mAbs that tested positive in ELISA against the short recombinant NY-BR-1-aa851928 that was used for immunization before, a polyclonal anti-GFP antibody (BD Biosciences, San Jose, CA), and polyclonal antibody to $\beta$-actin (Sigma Aldrich, St Louis, MO).

\section{Immunohistochemistry}

The newly generated hybridomas that showed specific reactivity with NY-BR-1 in preceding analyses were tested by immunohistochemistry as described previously. ${ }^{5-7}$ Culture media of hybridoma clones or antibodies purified from ascetic fluid of mice were used. Frozen and formalin-fixed paraffin-embedded tissue specimens were obtained from the archives of the New York Branch of the Ludwig Institute of Cancer Research, the Department of Pathology of Memorial Sloan-Kettering Cancer Center, and from the Laboratory of Surgical Oncology of Hadassah Hospital Jerusalem. All specimens were procured under the valid local legal regulations. To ensure the applicability of newly generated anti-NY-BR-1 mAbs to archival material, IHC analyses were commenced on standard archival formalin-fixed paraffinembedded tissues using hybridoma supernatants at different dilutions. A heat-based antigen-retrieval technique was used for antigen recovery on paraffin slides employing a household vegetable steamer (Oster, Boca Raton, FL) and various buffer solutions such as citrate buffer (10 mM, pH6.0), EDTA (1 mM, pH8.0), and DAKO hipH solution (DAKOCytomation, Carpinteria, CA). After deparaffinization, slides were heated for 30 minutes in the buffer solution before the IHC staining. Primary antibody incubation was done overnight at $4{ }^{\circ} \mathrm{C}$ followed by a biotinylated horse-anti-mouse secondary (1:200, Vector Labs, Burlingame, CA) and an avidinbiotin complex system (Vector Elite, Vector Labs). Diaminobenzidine (liquid DAB, BioGenex, San Ramon, CA) was used as a chromogen. IHC staining was estimated by microscopy as the percentage of stained cells and graded as follows: focal $<5 \% ;+5 \%$ to $25 \%$; $++>25 \%$ to $50 \% ;+++>50 \%$ to $75 \%$; and $++++>75 \%$. Hybridomas showing staining in paraffin specimens were also tested on frozen tissues. Supernatants of positive hybridoma clones were purified by protein $\mathrm{G}$ purification and further analyses were carried out using purified mAbs. The IHC reactivity pattern of the newly generated mAbs was then tested on normal and tumor tissues, including a panel of breast carcinomas, various nonbreast carcinomas, and a small series of sweat gland carcinomas. To analyze the loss of antigen in stored tissue sections, we also compared the immunostaining in slides that were from freshly cut 
paraffin bocks with slides to which tissue sections were applied in the past. Matched isotype controls using the same concentration as the test antibodies were used as negative controls.

\section{RESULTS}

Five newly generated clones, NY-BR-1 \#2, \#3, \#4, \#5, and \#6 showed reactivity in ELISA assays with the recombinant NY-BR-1 protein (aa 851-928) that was used to immunize the mice (data not shown). Those 5 clones were further tested for specificity by Western blotting. All 5 clones showed reactivity with NY-BR-1transfected 293 T cells (full-length NY-BR-1 aa 1-1397, and GFP-NY-BR-1 aa1-1397) and not with untransfected $293 \mathrm{~T}$ cells (Fig. 1) or $293 \mathrm{~T}$ cells transfected with the empty vector (analyzed by RT-PCR, data not shown). As shown in Figure 1, staining with the polyclonal antibody against GFP detected a similar band as the NY-BR-1 antibody. This demonstrated that the monoclonal antibody recognized the NY-BR-1 protein in lysates of NYBR-1-transfected cells and did not react with proteins derived from nontransfected cells. In addition, the newly generated mAbs were tested with recombinant protein preparations of NY-BR-1, as negative controls NY-ESO1, MAGE-A10, and SSX-4 recombinant proteins were used (Fig. 2). All 5 clones (\#2, \#3, \#4, \#5, and \#6) were then tested by immunohistochemistry.

The initial IHC analysis was done on formalin-fixed paraffin-embedded breast. Clones \#2, \#3, \#4, and \#5 showed staining in breast tissue comprising of immuno-

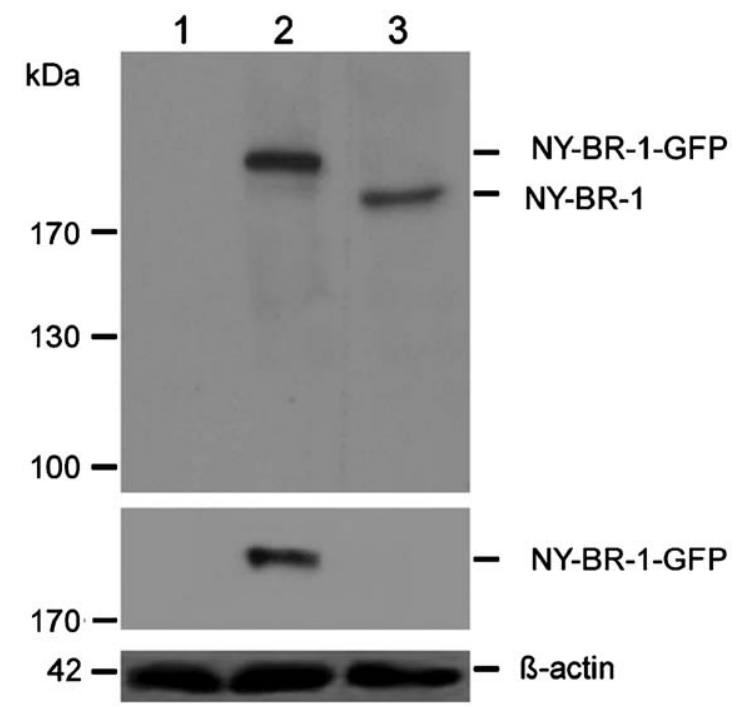

FIGURE 1. Immunoblot analysis of transiently transfected 293 T cells using newly generated mAb NY-BR-1\#2. Lysates from $293 \mathrm{~T}$ cells transiently expressing the indicated constructs were analyzed with an anti-NY-BR-1 antibody (upper panel, dilution 1:500) and anti-GFP antibody (middle panel). $\beta$-actin served as the loading control (lower panel). Lane 1, empty vector control; lane 2, pcDNA3.1-NY-BR-1-GFP; lane 3, pCDNA3.1-NY-BR-1.

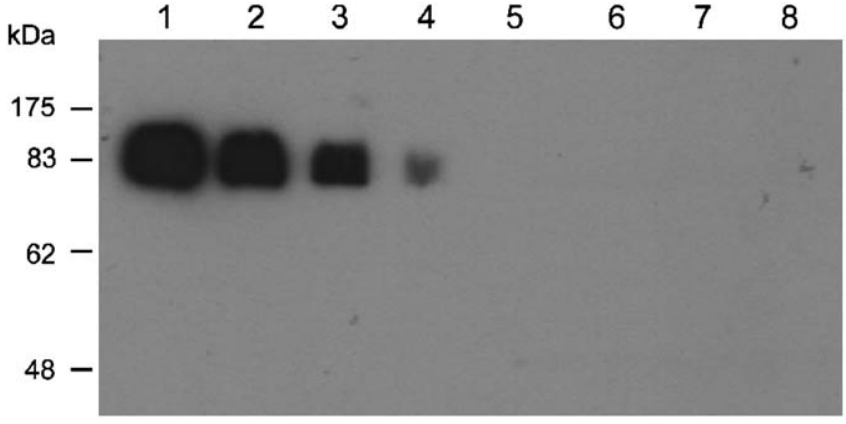

FIGURE 2. Different dilutions of anti-NY-BR-1 supernatants were tested against the recombinant NY-BR-1 protein (aa7011397 ) in Western blot (lanes 1 to 4 supernatant at 1:250, $1: 500,1: 1000$, and 1:2000 dilutions, 5 representing the negative control using buffer. The irrelevant recombinant proteins derived from NY-ESO-1, MAGE-A10, and SSX-4 (lanes 6,7 , and 8 ) were also used as negative controls).

positive ductal and lobular epithelial cells. Clones \#2 and \#3 gave the most intense staining with the least background staining. Both clones were purified, isotypes analyzed, and again titered on breast tissue and the staining using antigen retrieval was optimized. Both mAbs, NY-BR-1\#2 and NY-BR-1\#3 were IgG1(kappa) isotypes, and worked best with EDTA as antigen-retrieval solution revealing a similar staining pattern. NY-BR-1\#2 however, showed the best staining properties and could be used at a wide concentration range without revealing any unspecific immunoreactivity. Consequently, clone NY-BR-1\#2 was chosen for subsequent analyses. No reactivity was seen in frozen tissue with both mAbs.

To asses the NY-BR-1 expression pattern, a panel of normal tissues and various tumors were tested with mAb NY-BR-1\#2 by immunohistochemistry. In normal adult tissues, immunostaining was solely present in breast and no NY-BR-1\#2 immunoreactivity was visible in any other tissue (Table 1). Interestingly, among the negative tissues was testis, that showed expression of NY-BR-1 on an mRNA level. In mammary gland, the typical NY-BR$1 \# 2$ staining pattern showed single or smaller foci of

TABLE 1. IHC Staining With mAb NY-BR-1\#2 in Normal Tissues

\begin{tabular}{lc}
\hline Tissue & NY-BR-1\#2 \\
\hline Colon & - \\
Lung & - \\
Liver & - \\
Kidney & - \\
Testis & - \\
Mammary gland & Positive \\
Skin & - \\
Brain & - \\
Stomach & - \\
Pancreas & - \\
Small intestine & - \\
Placenta & - \\
\hline
\end{tabular}


immunopositive cells in lobules and ducts (Fig. 3). These positive cells consisted almost exclusively of epithelial ductal and lobular cells whereas myoepithelial cells typically remained negative. Most often, staining in lobules and ducts comprised foci or just single intensely immunopositive cells amongst negative cells (Figs. 3A, B, D). The cellular staining pattern was predominantly cytoplasmic and occasionally nuclear (Fig. 3B). In some tissues, however, areas were present in which most or all cells of a particular lobule or duct were immunopositive (Fig. 3C). And in some cases, these areas with focal and extensive NY-BR-1\#2 immunostaining could be observed side by side within the same tissue. More staining appeared to be present in normal epithelia of ducts and lobules next to in situ or invasive carcinoma areas. Some NY-BR-1\#2 staining could be seen in all normal breast tissue tested (Fig. 3 and Table 1).

To evaluate the presence of NY-BR-1 in tumors, various neoplasms were analyzed by immunohistochemistry (Tables 2 and 3). Logically, the expression pattern in breast carcinoma was of foremost interest. This was studied using 30 blocks derived from 20 cases of ductal
FIGURE 3. IHC staining with mAb NY-BR$1 \# 2$ in normal (A-D) and neoplastic $(F, G)$ breast tissue, testis (E), and mucinous sweat gland (eccrine) carcinoma $(H)$ (ABC method, DAB chromogen). (A) typical NY-BR-1\#2 staining in breast lobule, (B) single cells epithelial cells in acinus of breast lobule with cytoplasmic and nuclear staining, (C) intense staining of all epithelium in a single lobule, (D) focal NY-BR-1-positive ductal epithelium in a duct, (E) negative testicular tubule (F) intensely positive intraductal carcinoma cells, (G) moderately intense staining of invasive complexes of a breast carcinoma, and $(\mathrm{H})$ intense staining of neoplastic epithelium in a primary cutaneous sweat gland carcinoma.
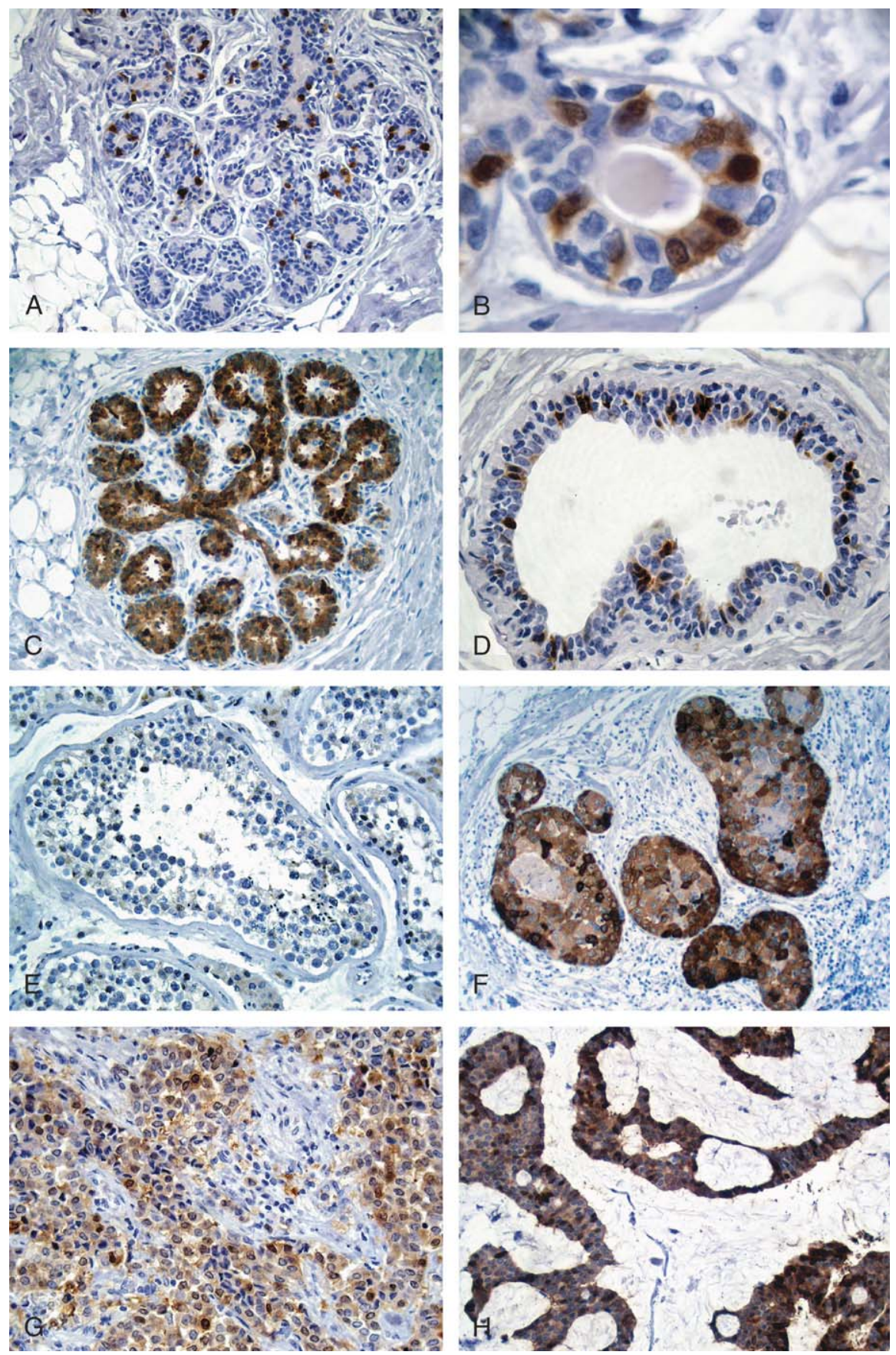

(C) 2007 Lippincott Williams \& Wilkins 
TABLE 2. IHC Staining With mAb NY-BR-1\#2 in 20 Ductal Breast Carcinomas

\begin{tabular}{lcc}
\hline NY-BR-1 & Carcinoma in Situ/Intraduct ca & Invasive Carcinoma \\
\hline Total & 11 & 10 \\
Neg. & 1 & 5 \\
Foc & 1 & - \\
+ & - & 2 \\
++ & - & - \\
+++ & 3 & 2 \\
+++++ & 6 & 1 \\
Total positive & $10 / 11$ & $5 / 10$ \\
\hline
\end{tabular}

carcinoma. In $11 / 20$ cases ductal carcinoma in situ (DCIS) and in 10/20 invasive ductal carcinoma was present. Of the 11 DCIS cases, 10/11 tumors were NYBR-1\#2 positive. Interestingly, many of the immunopositive (9/10) DCIS cases showed immunostaining in more than $50 \%$ of the intraductal carcinoma areas $(+++$ to ++++ according to our grading system). Surprisingly, in the 10 cases with invasive carcinoma, only $5 / 10$ were NY-BR-1 positive and a homogeneous expression pattern $(+++$ to ++++$)$ was present in $3 / 5$ NY-BR-1positive cases. Within the tumors, large differences in the staining intensities of the cells were visible. In general, DCIS seemed to display a more intense immunoreactivity than invasive carcinomas (Figs. 3F, G). An IgG1 isotype control antibody used at the same concentration as NYBR-1\#2 was negative in all tested breast specimens. Because of their similarity to breast carcinomas, 11 primary sweat gland tumors were also analyzed. Three of them were immunoreactive with NY-BR-1\#2. Two of the 3 positive sweat gland tumors were eccrine duct carcinoma, 1 was a primary cutaneous mucinous carcinoma. The latter showed strong and homogeneous staining thoughout the entire tumor.

Various other tumors such as carcinomas of the kidney (renal cell carcinoma), liver (hepatocellular

TABLE 3. Immunohistochemical Staining With mAb NY-BR$1 \# 2$ in Various Tumors

\begin{tabular}{lcl}
\hline Tissue & NY-BR-1\#2 \\
\hline Colon carcinoma & $0 / 10$ & \\
Non-small cell lung carcinoma & $0 / 40$ & \\
Hepatocellular carcinoma & $0 / 4$ & \\
Renal cell carcinoma (9 clear cell, & $0 / 11$ & \\
$\quad$ 1 chromophobe, 1 papillary) & & \\
Seminoma & $0 / 4$ & \\
Ovarian carcinoma (6 serous, & $0 / 9$ & \\
$\quad 3$ endometroid) & & \\
Malignant melanoma (38 metastatic, & $0 / 44$ & $(1 \times++++$, \\
$\quad$ 6 desmoplastic)* & & $1 \times+, 1 \times$ \\
Merkel cell carcinoma* & $0 / 40$ & focal) \\
Pancreatic carcinoma* & $0 / 18$ & \\
Sweat gland tumors & $3 / 11$ & \\
& & \\
& &
\end{tabular}

*Tissue microarray-based analysis. carcinoma), and adenocarcinoma of the colon as well as cutaneous melanoma and ovarian carcinoma were not immunoreactive with NY-BR-1\#2 (Table 3). Four cases of seminoma also remained negative.

As it is known that some antigens lose their detectability in immunohistochemistry after prolonged storage as cut sections on a slide, we evaluated the immunostaining of NY-BR-1 in normal breast sections, which had been cut and stored at room temperature 6 months or longer before their use. Best staining was achieved in freshly cut tissue sections, whereas the immunostaining deteriorated in older sections. One slide of normal breast tissues cut and stored 8 years ago, was almost negative although a freshly cut section from the same block revealed the characteristic NY-BR-1 expression pattern.

\section{DISCUSSION}

On the basis of their ability to elicit T-cell-mediated or B-cell-mediated immunoresponses in the autologous host, several novel tumor antigens or antigen families such as MAGE, GAGE, NY-ESO-1, CT7, and others have been isolated in the last decade. Although little is known about the biology and function of most of these antigens, their limited presence in normal tissues and their almost selective expression in tumors has made them a focus in the search for potential targets for the immunotherapy of cancer. ${ }^{8,9}$ Several clinical trials employing these antigens have been initiated, some with encouraging results. ${ }^{10,11}$ However, most of the molecularbased methods used for the identification of these antigens, render primary mRNA data, which allow rapid gene identification and almost instant expression analysis by RT-PCR, whereas protein expression studies require the comparably laborious generation of serologic reagents. However, the proper assessment of potential immunotherapy targets requires knowledge about the actual presence of the antigen on a protein level. ${ }^{12-14}$

NY-BR-1 was recently identified by an autologous serological typing approach in a breast carcinoma patient. ${ }^{1,2}$ Initial mRNA analysis indicated the presence of NY-BR-1 in normal mammary gland and neoplastic breast tissue and in testis. The present study was intended to generate serologic reagents for the analysis of NY-BR1 on a protein level. As formalin-fixed paraffin embedded tissues are available for many cancer patients, we adjusted our hybridoma screening protocols to ensure the selection of reagents applicable to standard archival material. As a consequence, 2 mAbs NY-BR-1\#2 and NY-BR-1\#3 have become available for the analysis of NY-BR-1 expression in archival paraffin blocks. The initial IHC analysis confirms previous mRNA data regarding the presence of NY-BR-1 in breast tissue and defines the actual location of NY-BR-1 expressing cells. No NY-BR-1 protein expression could be located to any other normal human tissues. Interestingly, previous RT-PCR analysis showed high levels of NY-BR-1 mRNA in testicular tissue. ${ }^{1,2}$ However, present IHC staining did not reveal any protein 
in testis. A discordance of mRNA and protein expression in testis has been shown for many genes and seems to be a consequence of translational control important for mammalian germ-cell development. ${ }^{1,2,15,16}$ The sole expression in normal and neoplastic breast tissues indicates that NY-BR-1 is a true differentiation antigen. Nevertheless, NY-BR-1 protein is present in human mammary gland epithelia in a very heterogeneous fashion and only few cells or small groups of epithelium were immunopositive in normal lobules or ducts. However, NY-BR-1positive cells, albeit occasionally very few, were present in all normal breast tissue areas that were analyzed. Interestingly, NY-BR-1 expression was most prevalent in DCIS. Interestingly, the expression pattern in DCIS was predominantly homogeneous and NY-BR-1 staining usually comprised most of the lesions. Surprisingly, in invasive carcinomas NY-BR-1 expression was less prevalent. Not only were fewer cases positive, the expression pattern was also heterogeneous comprising oftentimes only smaller parts of the tumor.

The restricted presence of NY-BR-1 protein in approximately $50 \%$ of the invasive carcinoma cases contrasted with previous RT-PCR data, which demonstrated NY-BR-1 mRNA in more than $80 \%$ of invasive breast tumors. ${ }^{1}$ The discordance between mRNA and protein expression is most likely due to the sample error. Asinvasive carcinoma specimens often contain normal mammary gland tissue and/or intraductal components, the presence of NY-BR-1 in normal breast tissue and DCIS will render those invasive carcinoma samples NYBR-1 mRNA positive. The ability to define clearly the NY-BR-1 expressing histologic components underlines the importance of generating serologic reagents for a comprehensive gene expression analysis. The present immunohistochemical data suggest that NY-BR-1 expression decreases with advancement of the disease. This is further supported by the homogeneous presence of NYBR-1 in intraductal carcinoma versus the more heterogeneous expression in invasive tumors. Moreover, we recognized a difference in staining intensities in DCIS versus invasive carcinoma. Although immunohistochemistry is at best, a semiquantitative method which does not permit exact assessment of protein quantities, the less intense NY-BR-1 immunostaining in invasive areas versus the intense reactivity in DCIS areas also points toward lower levels of NY-BR-1 in more advanced tumors. Since the present analysis is based on a limited number of tumors, a larger panel of breast tumors, primary and metastatic, including clinical data that need to be analyzed to explore the full expression pattern and significance of NY-BR-1 in tumors of the mammary gland. A tissue microarray-based study analyzing a high number of ductal as well as other histological types of breast carcinoma has already been completed and shows that the protein expression of NY-BR-1 is also present in cancers other than ductal (manuscript in print).

Another interesting aspect of NY-BR-1 is its almost exclusive protein expression in breast epithelium. In surgical pathology, differential diagnosis of metastatic lesions can be an arduous exercise and pinpointing a primary lesion oftentimes rests on IHC markers to delineate the tissue of origin and serologic reagents to differentiation markers are often used to locate the organ or tissue of origin. ${ }^{17,18}$ Examples are mAbs HMB45 and A103 to melanocyte differentiation antigens gp100 and Melan-A, respectively, for the diagnosis of melanoma or mAbs to CD45/LCA, an antigen expressed on hematopoetic cells, which aid in the diagnosis of leukemias and lymphomas. ${ }^{6,19,20}$ For the diagnosis of breast carcinomas, immunohistochemistry for hormone receptors are commonly used. However, expression of estrogen or progesterone receptors and other markers are not restricted to carcinomas of the mammary gland and has been observed in other tumors. ${ }^{21,22}$ Consequently, the restricted expression of NY-BR-1 could be of value as a diagnostic marker. However, in this context it needs to be considered that NY-BR-1 is present in sweat gland tumors too. This is not surprising because the mammary gland is a modified sweat gland and the expression of markers to breast epithelium in sweat gland tumors is well known for other antigens. ${ }^{23,24}$ Although this may only rarely pose a diagnostic dilemma, one should be aware of the potential presence of NY-BR-1 in sweat gland tumors once it is used as a diagnostic marker, especially when tumors of cutaneous appendages are suspected in the skin of the breast.

From a technical standpoint, it needs to be considered that NY-BR-1 immunohistochemistry should be carried out on slides that have been cut recently because the antigen seems to deteriorate when stored as a cut slide. Loss of antigenicity in stored slides is known for many other antigens in immunohistochemistry and obviously needs to be considered in NY-BR-1 protein expression analysis too. ${ }^{25-28}$

In conclusion, we present 2 new mAbs to NY-BR-1 including a preliminary IHC analysis in a limited number of normal tissues and tumors. Our data show that both reagents are reliable IHC markers and that NY-BR-1 expression is solely present in normal and neoplastic human breast epithelia. This expression pattern suggests that NY-BR-1 is a differentiation antigen of the human breast epithelium, which may be a valuable diagnostic marker and a potential target for the immunotherapy of breast carcinomas. Extensive IHC analyses in a high number of breast carcinomas are necessary and currently undertaken to characterize precisely the protein expression pattern of NY-BR-1.

\section{REFERENCES}

1. Jager D, Stockert E, Gure AO, et al. Identification of a tissuespecific putative transcription factor in breast tissue by serological screening of a breast cancer library. Cancer Res. 2001;61:2055-2061.

2. Jager $\mathrm{D}$, Unkelbach $\mathrm{M}$, Frei $\mathrm{C}$, et al. Identification of tumorrestricted antigens NY-BR-1, SCP-1, and a new cancer/testis-like antigen NW-BR-3 by serological screening of a testicular library with breast cancer serum. Cancer Immun. 2002;2:5.

3. Chen YT, Stockert E, Chen Y, et al. Identification of the MAGE-1 gene product by monoclonal and polyclonal antibodies. Proc Natl Acad Sci USA. 1994;91:1004-1008. 
4. Chen YT, Stockert E, Jungbluth A, et al. Serological analysis of Melan-A[MART-1], a melanocyte-specific protein homogeneously expressed in human melanomas. Proc Natl Acad Sci USA. 1996;93:5915-5919.

5. Jungbluth AA, Stockert E, Chen YT, et al. Monoclonal antibody MA454 reveals a heterogeneous expression pattern of MAGE-1 antigen in formalin-fixed paraffin embedded lung tumours. $\mathrm{Br}$ $J$ Cancer. 2000;83:493-497.

6. Jungbluth AA, Iversen $\mathrm{K}$, Coplan $\mathrm{K}$, et al. T311-an anti-tyrosinase monoclonal antibody for the detection of melanocytic lesions in paraffin embedded tissues. Pathol Res Pract. 2000; 196:235-242.

7. Jungbluth AA, Chen YT, Stockert E, et al. Immunohistochemical analysis of NY-ESO-1 antigen expression in normal and malignant human tissues. Int J Cancer. 2001;92:856-860.

8. Boon T, Old LJ. Cancer Tumor antigens. Curr Opin Immunol. 1997;9:681-683.

9. Scanlan MJ, Gure AO, Jungbluth AA, et al. Cancer/testis antigens: an expanding family of targets for cancer immunotherapy. Immunol Rev. 2002;188:22-32.

10. Davis ID, Chen W, Jackson H, et al. Recombinant NY-ESO-1 protein with ISCOMATRIX adjuvant induces broad integrated antibody and $\mathrm{CD} 4[+]$ and $\mathrm{CD} 8[+] \mathrm{T}$ cell responses in humans. Proc Natl Acad Sci USA. 2004;101:10697-10702.

11. van Baren N, Brasseur F, Godelaine D, et al. Genes encoding tumor-specific antigens are expressed in human myeloma cells. Blood. 1999;94:1156-1164.

12. Jager E, Stockert E, Zidianakis Z, et al. Humoral immune responses of cancer patients against "cancer-testis" antigen NYESO-1: correlation with clinical events. Int J Cancer. 1999; 84:506-510.

13. Knuth A, Jager D, Jager E. Cancer immunotherapy in clinical oncology. Cancer Chemother Pharmacol. 2000;46:46-51.

14. Jager D, Jager E, Knuth A. Vaccination for malignant melanoma: recent developments. Oncology. 2001;60:1-7.

15. Kleene KC. Patterns, mechanisms, and functions of translation regulation in mammalian spermatogenic cells. Cytogenet Genome Res. 2003;103:217-224.
16. Kleene KC. Sexual selection, genetic conflict, selfish genes, and the atypical patterns of gene expression in spermatogenic cells. Dev Biol. 2005;277:16-26.

17. Dennis JL, Hvidsten TR, Wit EC, et al. Markers of adenocarcinoma characteristic of the site of origin: development of a diagnostic algorithm. Clin Cancer Res. 2005;11:3766-3772.

18. Hammar SP, Orenstein JM. Metastatic neoplasms of unknown primary origin: an overview. Ultrastruct Pathol. 1992;16:3-5.

19. Kaufmann O, Koch S, Burghardt J, et al. Tyrosinase, melan-A, and KBA62 as markers for the immunohistochemical identification of metastatic amelanotic melanomas on paraffin sections. Mod Pathol. 1998; 11:740-746.

20. Jungbluth AA, Busam KJ, Gerald WL, et al. A103: An anti-melan-a monoclonal antibody for the detection of malignant melanoma in paraffin-embedded tissues. Am J Surg Pathol. 1998;22:595-602.

21. Dabbs DJ, Landreneau RJ, Liu Y, et al. Detection of estrogen receptor by immunohistochemistry in pulmonary adenocarcinoma. Ann Thorac Surg. 2002;73:403-405.

22. Dennis JL, Hvidsten TR, Wit EC, et al. Markers of adenocarcinoma characteristic of the site of origin: development of a diagnostic algorithm. Clin Cancer Res. 2005;11:3766-3772.

23. Busam KJ, Gellis S, Shimamura A, et al. Small cell sweat gland carcinoma in childhood. Am J Surg Pathol. 1998;22:215-220.

24. Kariya Y, Moriya T, Suzuki T, et al. Sex steroid hormone receptors in human skin appendage and its neoplasms. Endocr J. 2005; $52: 317-325$.

25. Bertheau P, Cazals-Hatem D, Meignin V, et al. Variability of immunohistochemical reactivity on stored paraffin slides. J Clin Pathol. 1998;51:370-374.

26. Fergenbaum JH, Garcia-Closas M, Hewitt SM, et al. Loss of antigenicity in stored sections of breast cancer tissue microarrays. Cancer Epidemiol Biomarkers Prev. 2004;13:667-672.

27. Jacobs TW, Prioleau JE, Stillman IE, et al. Loss of tumor markerimmunostaining intensity on stored paraffin slides of breast cancer. J Natl Cancer Inst. 1996;88:1054-1059.

28. Vis AN, Kranse R, Nigg AL, et al. Quantitative analysis of the decay of immunoreactivity in stored prostate needle biopsy sections. Am J Clin Pathol. 2000;113:369-373. 